\title{
LOFAR view of NGC 3998, a sputtering AGN ${ }^{\star}$
}

\author{
Sarrvesh S. Sridhar ${ }^{1,2}$, Raffaella Morganti ${ }^{1,2}$, Kristina Nyland ${ }^{3}$, Bradley S. Frank ${ }^{4,5}$ \\ , Jeremy Harwood ${ }^{6}$, and Tom Oosterloo ${ }^{1,2}$
}

\author{
1 Kapteyn Astronomical Institute, University of Groningen, PO Box 800, 9700 AV Groningen, The Netherlands \\ 2 ASTRON, The Netherlands Institute for Radio Astronomy, Postbus 2, 7990 AA Dwingeloo, The Netherlands \\ e-mail: morganti@astron.nl \\ 3 National Research Council, Resident at the U.S. Naval Research Laboratory, 4555 Overlook Ave. SW, Washington, DC 20375, \\ USA \\ 4 South African Radio Astronomical Observatory (SARAO), 2 Fir Street, Observatory 7925, South Africa \\ 5 Department of Astronomy, University of Cape Town, Private Bag X3, Rondebosch 7701, South Africa \\ ${ }^{6}$ Centre for Astrophysics Research, School of Physics, Astronomy and Mathematics, University of Hertfordshire, College Lane, \\ Hatfield AL10 9AB, UK
}

Received 27 September 2019 / Accepted 27 November 2019

\begin{abstract}
Low-power radio sources dominate the radio sky. They tend to be small in size and dominated by their cores, but the origin of their properties and the evolution of their radio plasma are not well constrained. Interestingly, there is mounting evidence that low-power radio sources can significantly affect their surrounding gaseous medium and may therefore be more relevant for galaxy evolution than previously thought. In this paper, we present low radio frequency observations obtained with LOFAR at $147 \mathrm{MHz}$ of the radio source hosted by NGC 3998. This is a rare example of a low-power source that is extremely dominated by its core, but that has two largescale lobes of low surface brightness. We combine the new $147 \mathrm{MHz}$ image with available $1400 \mathrm{MHz}$ data to derive the spectral index over the source. Despite the low surface brightness, reminiscent of remnant structures, the lobes show an optically thin synchrotron spectral index $(\sim 0.6)$. We interpret this as being due to rapid decollimation of the jets close to the core, to high turbulence of the plasma flow, and to entrainment of thermal gas. This could be the result of intermittent activity of the central active galactic nucleus, or, more likely, temporary disruption of the jet due to the interaction of the jet with the rich circumnuclear interstellar matter. Both would result in sputtering energy injection from the core, which would keep the lobes fed, albeit at a low rate. We discuss these results in connection with the properties of low-power radio sources in general. Our findings show that amorphous low surface brightness lobes should not be interpreted by default as remnant structures. Large deep surveys (in particular the LOFAR $150 \mathrm{MHz}$ LoTSS and the recently started $1400 \mathrm{MHz}$ Apertif survey) will identify a growing number of objects similar to NGC 3998 where these ideas can be further tested.
\end{abstract}

Key words. galaxies: active - radio continuum: galaxies - galaxies: individual: NGC 3998 - ISM: jets and outflows

\section{Introduction}

One of the key ingredients that all cosmological simulations agree is required to reproduce the observed properties of massive galaxies, is the (self-regulating) process that links the enormous amount of energy that is released by an active super massive black hole (i.e. active galactic nucleus, AGN) to the surrounding interstellar (ISM) and intergalactic (IGM) medium (see Heckman \& Best 2014 and references therein). Because it is important, but also because it is complex, great effort (in observations and theory) has been made and still continues to constrain its properties.

In the past years, evidence has been mounting about the relevance of, among others, low-power radio sources for this process. Low-power radio sources $\left(\leq 10^{24} \mathrm{~W} \mathrm{~Hz}^{-1}\right)$ represent a class of relatively common AGN, being observed in at least $30 \%$ of massive early-type galaxies (i.e. $M_{*}>10^{11} M_{\odot}$, Mauch \& Sadler 2007; Best et al. 2005; Sabater et al. 2019). Their occurrence in

* The reduced images are only available at the CDS via anonymous ftp to cdsarc.u-strasbg.fr (130.79.128.5) or via http: //cdsarc.u-strasbg.fr/viz-bin/cat/J/A+A/634/A108 massive galaxies is much higher than that of powerful radio AGN.

One of the relevant parameters that define the effect on the host galaxy is the duty cycle of the activity (see e.g. Ciotti et al. 2010; Gaspari et al. 2013; Morganti 2017). Interestingly, lowpower radio sources hosted by massive elliptical galaxies have been suggested to be "on" most of the time, with only short intervals of no activity. This means that they almost continuously dump energy on the host galaxy (Sabater et al. 2019). However, this has not yet been confirmed by detailed studies of radio AGN (see e.g. Nyland et al. 2016). Finally, a growing number of observations has shown that the gas in the central regions can indeed also be affected by low-power jets (Croston et al. 2008; Combes et al. 2013; Morganti et al. 2015; Rodríguez-Ardila et al. 2017; Fabbiano et al. 2018; Husemann et al. 2019 and references therein), and numerical simulations predict that this should occur (Mukherjee et al. 2018a,b). It is therefore important to consider the effect of this class of objects, which represents the most common population of radio AGN in the nearby Universe.

The morphology and properties of the radio emission appears to change with decreasing radio powers. At low radio 
power (below $\sim 10^{23}-10^{24} \mathrm{~W} \mathrm{~Hz}^{-1}$ ), the radio emission is often more compact (e.g. a few kiloparsec or lower) than the extent of the host galaxy, and it is more core dominated (see e.g. Balmaverde \& Capetti 2006; Baldi \& Capetti 2009; Baldi et al. 2019). The radio morphology of these objects could be the result of different physical properties or different evolutionary paths. For example, low-power sources tend to have low-velocity jets, and as a result of this, their jets are characterised by a larger component of turbulence than jets in powerful radio sources. This has been derived from detailed modelling of jets in FanaroffRiley type I (FRI, Fanaroff \& Riley 1974) radio galaxies (see e.g. Laing \& Bridle 2014; Massaglia et al. 2016), and these characteristics are expected to be even more extreme in jets with lower power, which have lower bulk Lorentz factors (Baldi et al. 2019). The turbulence and the consequent entrainment of the external thermal medium gives rise to high thermal fraction inside these jets, which causes them to slow down further (e.g. Bicknell et al. 1998).

Low-luminosity radio AGN have been suggested to be fuelled by a variety of mechanisms (see Storchi-Bergmann \& Schnorr-Müller 2019 for a review). This includes hot gas in advection-dominated accretion flows (Yuan \& Narayan 2014 and references therein), chaotic cold accretion (Gaspari et al. 2013, 2015) from gas cooling from the hot halo (e.g. Negri et al. 2015), and secular processes. The availability of this gas as fuel allows the nuclear activity to be restarted after only a short interruption, thus supporting recurrent nature of the nuclear activity with a high duty cycle.

Because of these physical properties and their galactic-scale jets, low-luminosity radio galaxies are also particularly sensitive to the external conditions. Numerical simulations show that the coupling between energy released by the jet and the ISM also remains high for low-power jets (e.g. Mukherjee et al. 2018a,b). Because they are less efficient in accelerating clouds, these jets are trapped in the ISM for a longer period of time, which increases the effect they can have on the ISM. This further suggests that these objects could play a role in the evolution of the host galaxy. Because of this, it is important to understand more about their life and the properties of the their jets and how they evolve.

We address this by studying one of these objects, the radio source hosted by NGC $3998^{1}$, which provides a unique case study. NGC 3998 represents one of the rare examples of a lowpower radio AGN $\left(P_{1400 \mathrm{MHz}} \sim 10^{22} \mathrm{~W} \mathrm{~Hz}^{-1}\right)$ with extended radio emission $(\sim 20 \mathrm{kpc})^{2}$. Here we expand the study presented in Frank et al. (2016) and focus on the morphology at low frequency $(147 \mathrm{MHz})$, and in combination with the previously published $1400 \mathrm{MHz}$ image, the radio spectral index. This information allows us to place new constraints on the age and evolution of the radio emission.

The paper is structured in the following way. In Sect. 2 we describe the properties of the target of our case study, NGC 3998 and its associated radio source. In Sect. 3 we present our new Low Frequency Array (LOFAR) observations. We discuss the results, including the spectral index distribution, in Sect. 4. Section 5 is dedicated to discussing the nature of the radio emission and the implications for other groups of low-power radio sources. We present our conclusions in Sect. 6.

\footnotetext{
1 We refer to it by the name of its optical identification.

2 We assume a distance of of $13.7 \mathrm{Mpc}(z=0.0035)$ for NGC 3998 (Cappellari et al. 2011). At this distance, $1^{\prime \prime}$ corresponds to $65.9 \mathrm{pc}$.
}

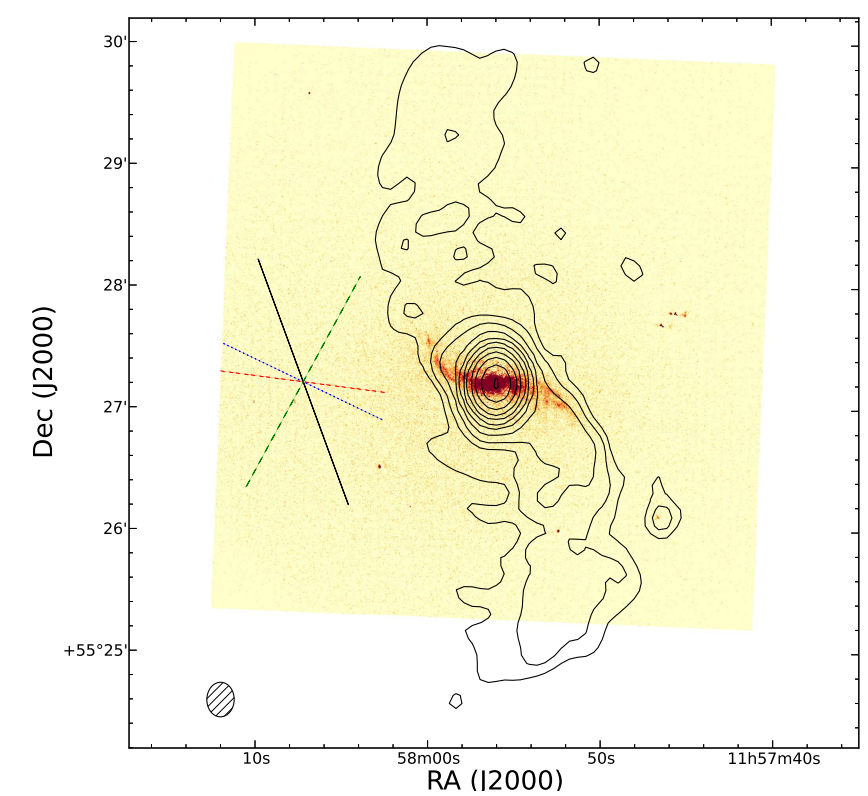

Fig. 1. Radio continuum contours from the narrow-band $21 \mathrm{~cm}$ observations of NGC 3998 using the Westerbork Synthesis Radio Telescope overlaid on the continuum-subtracted $\mathrm{H} \alpha$ image from Sánchez-Gallego et al. (2012). On the left, we show a depiction of the position angles of the various galaxy components. The green dashed line represents stars $\left(\mathrm{PA} \sim 135^{\circ}\right.$ from Krajnović et al. 2011), the black solid line the inner $\sim 5 \mathrm{kpc}$ of the radio continuum $\left(\mathrm{PA} \sim 33^{\circ}\right.$ ), the blue dotted line the $\mathrm{HI}$ disc $\left(\mathrm{PA} \sim 65^{\circ}\right)$, and red dot-dashed line the $\mathrm{H} \alpha$ disc $\left(\mathrm{PA} \sim 84^{\circ}\right)$. The image is taken from Frank et al. (2016).

\section{The target: NGC 3998}

As described in Frank et al. (2016), the radio source in NGC 3998 shows two S-shaped lobes (see Fig. 1) of low surface brightness $\left(\sim 5 \mathrm{mJy} \operatorname{arcmin}^{-2}\right.$ at $\left.1400 \mathrm{MHz}\right)$. In addition, a bright central component is also observed. This means that the radio source is characterised by an extremely high core dominance $\left(S_{\text {core }} / S_{\text {ext }}=8\right.$ or $S_{\text {core }} / S_{\text {tot }}=0.9$, derived at $1400 \mathrm{MHz}$, see Frank et al. 2016), much higher than is typically found for FRIs (Morganti et al. 1997; Laing \& Bridle 2014) and in the radio sources of lower power studied by Baldi et al. (2015). From a morphological point of view, the characteristics of the NGC 3998 lobes may suggest that they are still actively being fed by the nucleus (see Frank et al. 2016). However, structures of low surface brightness have often been suggested to be associated with remnants and are thought to no longer be fed by active jets (e.g. Saripalli et al. 2012; Brienza et al. 2016).

NGC 3998 is part of the ATLAS ${ }^{3 \mathrm{D}}$ sample (Cappellari et al. 2011) of nearby early-type galaxies. The radio study of this sample (e.g. Serra et al. 2012; Nyland et al. 2016, 2017) indicated that the type of radio structure found in NGC 3998 tends to be rare in low-power radio sources. Only 3 objects out of more than 100 for which deep continuum images are available (either at low or high resolution; Serra et al. 2012; Nyland et al. 2016) were found to have extended emission on a scale of a few dozen kiloparsec; one of these is NGC 3998 (the other two are NGC 3665 and NGC 5322).

NGC 3998 is a gas-rich early-type galaxy. Atomic neutral hydrogen was observed in this galaxy to be distributed in a nearly polar disc structure that extends over $10 \mathrm{Kpc}$ and has a mass of $\left(M_{\mathrm{HI}}=4 \times 10^{8} M_{\odot}\right)($ Serra et al. 2012; Frank et al. 2016). In ionised gas, Devereux (2018) reported the detection of broad permitted and forbidden emission lines using the high spatial 
resolution of the Space Telescope Imaging Spectrograph (STIS) on board the Hubble Space Telescope (HST). The authors suggested kinematically disturbed gas that is affected by the AGN emission. On larger scales, the $\mathrm{S}$-shaped radio lobes mirror the warp of the gas ( $\mathrm{HI}$ and $\mathrm{H} \alpha$ ), as illustrated in Fig. 1. This has led to the suggestion that in the inner kiloparsec regions of the galaxy, the stellar torques may be causing the gas disc to warp into an S-shape. These torques can also produce orbit crossings and shocks in the gas, which causes the gas to lose angular momentum, to accrete onto a central black hole and fuel the AGN. Thus, the fuelling of the central super-massive black hole might occur through accretion of cold gas through "discrete events", as also suggested by the observed variability of the radio core (Frank et al. 2016). However, an optically thin hot accretion flow is also a possibility, as shown by data from the Nuclear Spectroscopic Telescope Array (NUSTAR) and XMM-Newton X-ray telescope (Younes et al. 2019).

All of these properties mean that NGC 3998 is particularly well suited as a case study for understanding the evolution of the radio plasma in low-power AGN. One way to explore the evolution of these sources is to assess the spectral properties of their radio lobes. We perform this type of analysis for NGC 3998 using low-frequency observations at $147 \mathrm{MHz}$ carried out with the LOFAR (van Haarlem et al. 2013) combined with available data at $1400 \mathrm{MHz}$ presented in Frank et al. (2016).

\section{LOFAR observation and data reduction}

NGC 3998 was observed for eight hours with the LOFAR High Band Antenna (HBA) on 23 March 2015 (Open Time project LC3_025 PI K. Nyland). The eight-hour scan on NGC 3998 was bracketed with two $15 \mathrm{~min}$ scans on the flux density calibrator 3C 295. An overview of the observational parameters is presented in Table 1. The target and calibrator were observed with an identical frequency setup covering a bandwidth ranging from 123.7 MHz to $172.2 \mathrm{MHz}$. The frequency range was divided into $248195.3125 \mathrm{kHz}$ wide sub-bands (SBs) that were further subdivided into 64 channels each.

After the observation, the raw visibilities were first flagged for radio frequency interference (RFI) before they were averaged down to a frequency resolution of four channels per SB and $2 \mathrm{~s}$ time resolution. RFI flagging and averaging were carried out within the standard LOFAR pre-processing pipeline framework (Heald et al. 2010), which uses AOFlagger (Offringa et al. 2010, 2012) for RFI-flagging. Due to the large size of the raw dataset, only the averaged data products were uploaded to the LOFAR Long Term Archive (LTA) ${ }^{3}$. After pre-processing, the data were calibrated and imaged using the facet calibration scheme described in van Weeren et al. (2016) and Williams et al. (2016), which carries out calibration in two stages: a direction independent step followed by a direction-dependent calibration procedure.

In the direction-independent step, we first removed bad stations (CS013HBA0 and CS013HBA1) before we averaged the calibrator measurement sets to $1 \mathrm{ch} / \mathrm{sb}$ and $8 \mathrm{~s}$ time resolution. Using the Black Board Self-calibration software package (BBS, Pandey et al. 2009), we obtained amplitude and phase solutions for the RR and LL correlations using the 3C 295 model assuming the flux scale defined in Scaife \& Heald (2012). From the gain solutions, we computed the clock offset and gain amplitude for each LOFAR station and transferred these to the target

https://lta.lofar.eu
Table 1. LOFAR HBA observational parameters.

\begin{tabular}{ll}
\hline \hline Parameter & Value \\
\hline Project ID & LC3_025 \\
Observation ID & 294275 (3C 295) \\
& 294271 (NGC 3998) \\
Pointing centres & 14:11:20.5 +52:12:09.9 (3C 295) \\
& 11:57:56.1 +55:27:12.9 (NGC 3998) \\
Total on-source time & $8.0 \mathrm{~h}$ \\
Observation date & 2015 March 23 \\
Frequency range & 123.7-172.2 MHz \\
SBs & 248 SBs \\
Bandwidth per SB & 195.3125 KHz \\
LOFAR array mode & HBA Dual Inner \\
Stations & 61 total \\
\hline
\end{tabular}

measurement sets using BBS. Phase calibration was then applied to the clock- and gain-amplitude corrected target data using a $6 \times 6 \mathrm{deg}^{2}$ model of the sky from the TIFR GMRT Sky Survey (TGSS ADR1, Intema et al. 2017). The extracted skymodel contains 1504 point and 1144 Gaussian components.

The aim of the direction-dependent calibration procedure is to minimise the artefacts produced by the time- and positiondependent errors due to the ionosphere and the LOFAR station beam. To achieve this, we chose 15 directions or facets such that each facet contained at least one point source brighter than $750 \mathrm{mJy}$. For each facet, we performed self-calibration to derive good calibration solutions and models of the sources in that direction. Using the new calibration solutions, we then subtracted the sources in each of the 15 facets. Finally, the target facet containing NGC 3998 was corrected with calibration solutions derived using the bright source VLSS J1156.5+5520, which is $\sim 13^{\prime}$ away from NGC 3998 .

The direction-dependent calibrated visibilities were imaged using the wide-band deconvolution algorithm available in WSClean (Offringa et al. 2014) imager. We produced images of NGC 3998 at two different angular resolutions. The highresolution image was produced by weighting the visibilities using the Briggs weighting scheme with robust $=-0.5$ (Briggs 1995) and applying a 5" Gaussian taper. The restored image has an angular resolution of $11^{\prime \prime} .6 \times 7 ! \cdot 7$, and the rms noise close to NGC 3998 is about $170 \mu \mathrm{Jy}_{\text {beam }}{ }^{-1}$. To study the low surface brightness emission from the radio lobes, we also produced a low-resolution image using robust $=-0.7$ and applying a 25" Gaussian taper. The restored image was convolved with a $30^{\prime \prime}$ circular Gaussian beam, and the rms noise in the image is $680 \mu \mathrm{Jy}^{\text {beam }^{-1} \text {. }}$

We compared the integrated flux densities of background sources within our field of view with the flux densities reported in the TGSS ADR1 data release. We do not find any systematic offset in the integrated flux densities. We assume a $15 \%$ uncertainty for all the $147 \mathrm{MHz}$ flux densities reported below.

One of the goals of this work is to derive the spectral index of the various structures of the source, that is, nuclear region and lobes. We have done this using the integrated flux densities and by constructing a spectral index image using the LOFAR and the Westerbork Synthesis Radio Telescope (WSRT) from Frank et al. (2016). The spectral index $\alpha$ is defined as $S \sim v^{-\alpha}$. The integrated flux densities are derived over boxes including these structures, and they are summarised in Table 2. 
Table 2. Integrated flux densities of NGC 3998 taken from this paper and from Frank et al. (2016).

\begin{tabular}{|c|c|c|}
\hline $\begin{array}{l}\text { Freq } \\
(\mathrm{MHz})\end{array}$ & $\begin{array}{r}\text { Resolution } \\
(\operatorname{arcsec})\end{array}$ & $\begin{array}{r}\text { Flux density } \\
(\mathrm{mJy})\end{array}$ \\
\hline \multicolumn{3}{|l|}{ Core $^{(a)}$} \\
\hline $147 \mathrm{MHz}$ & 5 & $68 \pm 10$ \\
\hline $147 \mathrm{MHz}$ & 30 & $94 \pm 14$ \\
\hline $1400 \mathrm{MHz}$ & 15 & $149 \pm 7$ \\
\hline $4901 \mathrm{MHz}$ & 5 & $118 \pm 6$ \\
\hline \multicolumn{3}{|l|}{ Lobes $^{(b)}$} \\
\hline $147 \mathrm{MHz} \mathrm{N}$ & 30 & $31.3 \pm 7.6$ \\
\hline $147 \mathrm{MHz}$ S & 30 & $41.4 \pm 8.6$ \\
\hline $1400 \mathrm{MHz} \mathrm{N}$ & 30 & $8.0 \pm 1.1$ \\
\hline $1400 \mathrm{MHz} \mathrm{S}$ & 30 & $12.2 \pm 1.4$ \\
\hline
\end{tabular}

Notes. ${ }^{(a)}$ Peak flux density. The 147,1381 , and $4901 \mathrm{MHz}$ flux densities were recorded on 23 March 2015, 2 June 2015, and 20 June 2015, respectively ${ }^{(b)}$ Integrated in the same box - excluding the central regionat the two frequencies.

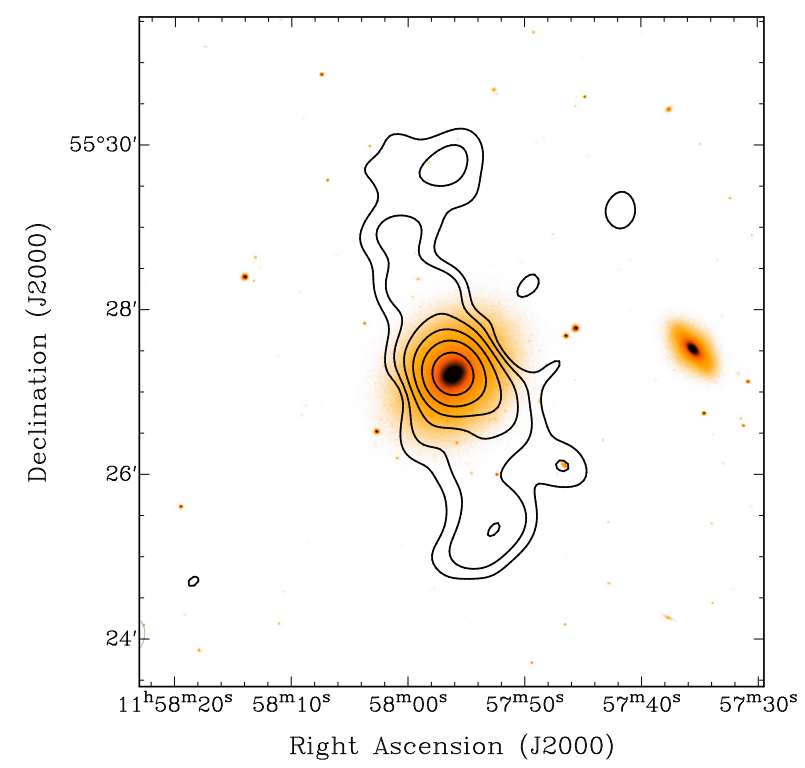

Fig. 2. Overlay of the $30^{\prime \prime}$ resolution Stokes I image of NGC 3998 (contours) and an $r$-band optical image from the Sloan Digital Sky Survey (Alam et al. 2015). The LOFAR contours are drawn at -1.8 and 1.8 to $94 \mathrm{mJy}^{\text {beam }}{ }^{-1}$ in steps of 2 .

\section{Results}

\subsection{NGC 3998 at low radio frequency}

Figure 2 shows the low-resolution image of NGC 3998 where the LOFAR total intensity contours are overlaid on an optical $r$-band image from the Sloan Digital Sky Survey (Alam et al. 2015). Our LOFAR image reveals the same S-shaped radio lobes seen in the WSRT $1400 \mathrm{MHz}$ radio images from Frank et al. (2016). The LOFAR image at higher spatial resolution (although still affected by artefacts, which are likely due to unmodelled ionospheric effects) allows us to better explore the nuclear regions. Figure 3 shows the high-resolution image of NGC 3998 superposed onto the low-resolution image.

The high-resolution image shows that the inner structure has, in addition to the bright core, a small extension to the $\mathrm{N}$ and a much clearer extension in the SW direction. The latter

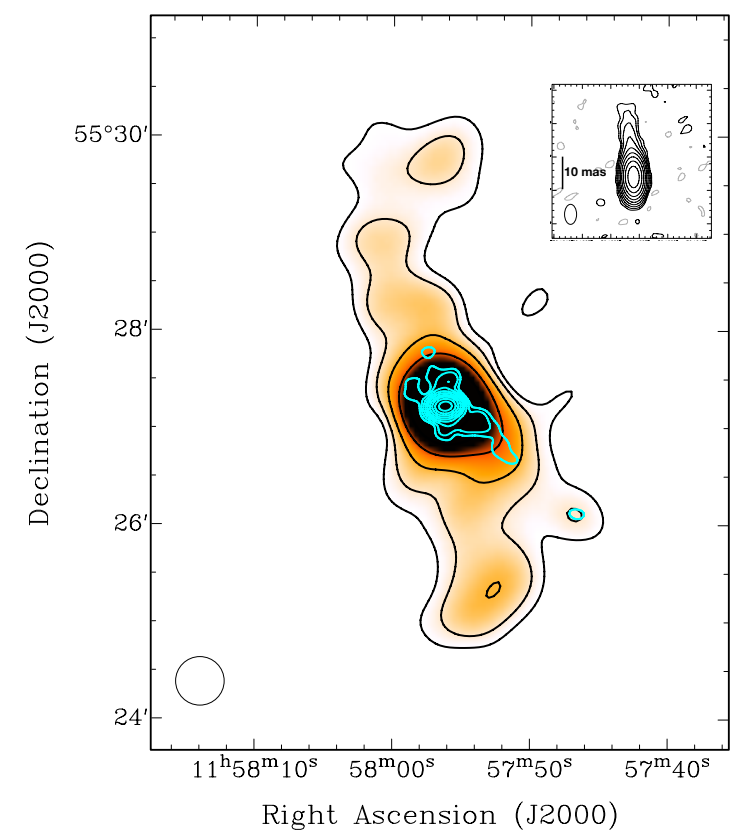

Fig. 3. LOFAR image ( $30^{\prime \prime}$ resolution). The contours of the highresolution LOFAR image are superposed. Contour levels are from -1.8 and 1.8 to $94 \mathrm{mJy}^{-1} \mathrm{beam}^{-1}$ in multiples of 2 and -1.8 and 1.8 to $68 \mathrm{mJy}$ beam $^{-1}$ in steps of 1.5 , respectively. The inset in the top right corner represents the VLBA image from Helmboldt et al. (2007) with contour levels of -0.5 and 0.5 to $200 \mathrm{mJy} \mathrm{beam}^{-1}$ in steps of 2 . The size of the VLBA jet is about 15 mas, corresponding to about $1 \mathrm{pc}$.

follows the structure seen in the WSRT image at $1400 \mathrm{MHz}$ well. Interestingly, on the very long baseline (VLBI) scale, the source shows a very small - about $1 \mathrm{pc}$ - one-sided jet elongated in the northern direction (Helmboldt et al. 2007), shown in the inset of Fig. 3. Thus, the jets in NGC 3998 appears to change direction while expanding in the inner kiloparsec regions. This may be due to precession, perhaps connected to the warping structure seen inside the inner regions (Frank et al. 2016). It is difficult to establish with the available data whether this change in the direction of the jet can be induced by interaction with the circumnuclear medium, however. At larger distances the jet lobes continues to warp, producing the S-shape structure.

The high core dominance, although not as extreme as at $1400 \mathrm{MHz}$, is also confirmed in the LOFAR image at $147 \mathrm{MHz}$. We derive $S_{\text {core }} / S_{\text {tot }}=0.32$, using the peak of the emission in the high-resolution image as core flux density.

\subsection{Spectral index distribution}

The spectral index image was constructed, following the procedure described in Harwood et al. (2013), using the $147 \mathrm{MHz}$ and the WSRT $1400 \mathrm{MHz}$, with matched uv range $(0.28-13.3 \mathrm{k} \lambda)$. The radio continuum images were convolved to a common resolution of $30^{\prime \prime}$ and placed on the same coordinate grid. All pixels below $5 \sigma_{\text {rms }}$ were masked and the spectral index error per pixel was estimated using the relation

$\alpha_{\text {err }}=\frac{1}{\log \frac{v_{\text {lofar }}}{v_{\text {wstr }}}} \sqrt{\left(\frac{S_{\text {lofar,err }}}{S_{\text {lofar }}}\right)^{2}+\left(\frac{S_{\text {wsrt,err }}}{S_{\text {wsrt }}}\right)^{2}}$,

where $S_{\text {lofar }}$ and $S_{\text {wsrt }}$ are the pixel values in the LOFAR and the WSRT maps, and $S_{\text {lofar,err }}$ and $S_{\text {wsrt,err }}$ are the uncertainties on 

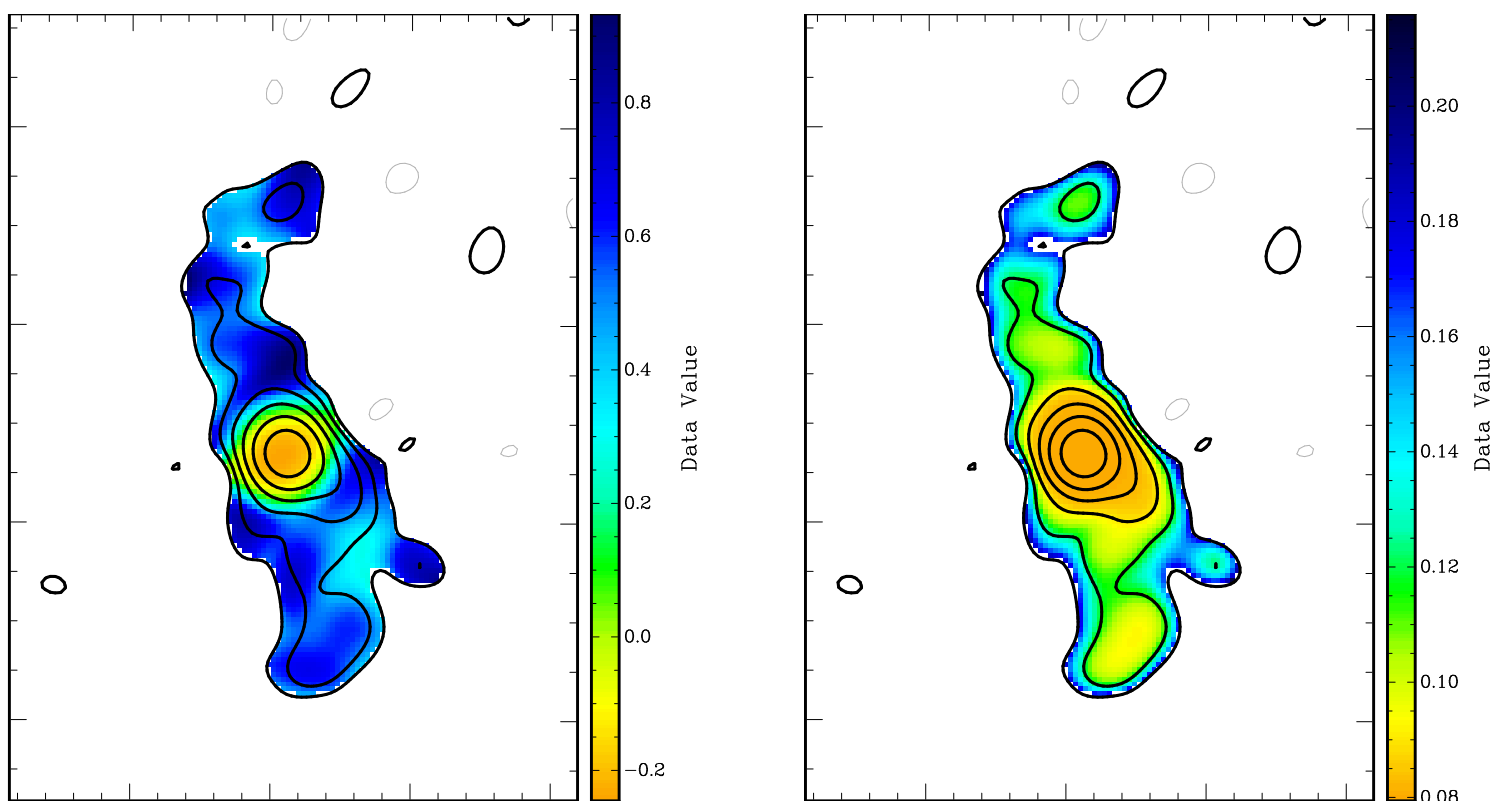

Fig. 4. Spectral index image (left) and associated error (right) between $147 \mathrm{MHz}$ and $1400 \mathrm{MHz}$, with superimposed contours as in Fig. 2.

each pixel value. Flux density calibration errors of $5 \%$ and $15 \%$ were assumed for WSRT and LOFAR, respectively.

Because the nuclear regions are variable, as discussed in (Frank et al. 2016), our observations must be used with care when the spectral index of the nuclear region is to be derived. In principle, simultaneous observations are required, or at least observations that are close in time.

Figure 4 shows the distribution of the spectral index (left) across the radio source between $147 \mathrm{MHz}$ and $1400 \mathrm{MHz}$ and the corresponding errors (right). The main result is that the spectral index of the low surface brightness lobes is typical of active lobes of radio galaxies with an average value of $\alpha_{147 \mathrm{MHz}}^{1400 \mathrm{MH}} \sim 0.6$. Figure 4 shows that the spectral index ranges between 0.4 and 0.8 . Furthermore, we see no trend in the spectral index moving away from the core. A trend (i.e. steepening of the spectral index with the distance from the core) is often seen in FRI with tailed structures, as described, for example, in Parma et al. (2002). This suggests that the conditions in NGC 3998 are different from those observed in radio galaxies where the spectral shape is dominated by the energy losses due to synchrotron emission. The presence of turbulence, which induces a strong mixing of the electron populations, could be at the origin of the relatively homogeneous spectral index seen in the lobes of NGC 3998 (see Sect. 5). An inverted spectrum $\left(\alpha_{147 \mathrm{MHz}}^{1400 \mathrm{MHz}} \sim-0.23 \pm 0.10\right)$ is instead clearly observed in the central region.

The spectral indices of the lobes and of the nuclear region are also confirmed by the results from the integrated spectral indices (see Table 2). The integrated flux densities of the lobes are derived from rectangular regions excluding the central component, while for the central region, the spectral indices could be derived from the higher resolution images and in two frequency ranges. For the central region, an inverted spectral index is confirmed at low frequencies $\left(\alpha_{147 \mathrm{MHz}}^{1400 \mathrm{MHz}} \sim-0.35 \pm 0.02\right)$, turning over at higher frequencies $\left(\alpha_{1400 \mathrm{MHz}}^{4800 \mathrm{MHz}} \sim+0.18 \pm 0.12\right)$, see Table 2. The flatter spectral index obtained at low frequencies compared to what the image of Fig. 4 shows is likely due to the lower spatial resolution of the latter (i.e. where the peak flux density at the core location include a larger portion from the beginning of the jet).
As mentioned above, we need to be careful about interpreting the exact value of the spectral index in the central regions because of the variability noted in the flux density and because the observations are not simultaneous (although quite close in time, see the notes to Table 2). The flux densities obtained from the $1400 \mathrm{MHz}$ and $4900 \mathrm{MHz}$ data were taken only three months apart; this is the closest we have in time (see Fig. 3 in Frank et al. 2016).

The overall spectrum of the nuclear regions seems to be consistent with a flat or self-absorbed spectrum, however, as expected if the central region is dominated by a very small source, $<2^{\prime \prime}(100 \mathrm{pc}$, as suggested by the work of Helmboldt et al. 2007). However, it is interesting to note that the derived slopes are not particularly steep and the spectral shape of the nuclear regions appears to be similar to what has been derived for FR0 radio sources (see Capetti et al. (2019)). The self-absorbed and bright core suggests that the core is the base of a relativistic synchrotron jet seen in powerful young radio galaxies.

\section{Nature of the radio emission in NGC 3998}

The main characteristics of the radio emission in NGC 3998 can be summarised as follows: The $147 \mathrm{MHz}$ LOFAR image shows two low-surface brightness lobes $\left(\sim 13 \mathrm{mJy} \mathrm{arcmin}^{-2}\right.$ at $147 \mathrm{MHz}$ ) with a similar extent as in the $1400 \mathrm{MHz}$ radio image by Frank et al. (2016). The lobes are reminiscent of plumes seen in FRI tailed radio galaxies (although typically at larger distances from the core). They also show an S-morphology that appears to follow the warped structure of the inner gas. This could also be reminiscent of cases of precessing jets (e.g. Kharb et al. 2006, 2010). The high-resolution LOFAR image traces the starting of the jets in the nuclear regions (see Fig. 3). The dominance of the nuclear region compared to the extended lobes is confirmed at low frequencies.

It is interesting to note that despite the low surface brightness, the spectral analysis suggests that the lobes are not old remnant structures. The integrated spectral indices of the lobes between $147 \mathrm{MHz}$ and $1400 \mathrm{MHz}$ are found to be around 
Table 3. Integrated spectral indices of NGC 3998 derived from the flux densities of Table 2.

\begin{tabular}{lcc}
\hline \hline \multirow{2}{*}{ Region } & \multicolumn{2}{c}{$\alpha$} \\
\cline { 2 - 3 } & $1400 \mathrm{MHz}$ & $\begin{array}{c}4900 \mathrm{MHz} \\
1400 \mathrm{MHz}\end{array}$ \\
\hline Core & $-0.35 \pm 0.02$ & $+0.18 \pm 0.12$ \\
N lobe & $+0.60 \pm 0.07$ & \\
S lobe & $+0.54 \pm 0.05$ & \\
\hline
\end{tabular}

$\alpha_{147 \mathrm{MHz}}^{1400 \mathrm{MHz}} \sim 0.6$ (see Fig. 4 and Table 3), consistent with values that are expected at these frequencies in active radio sources (see e.g. Heesen et al. 2018). These values are also consistent with no major steepening occurring at frequencies lower than $1400 \mathrm{MHz}$. Furthermore, the spectral indices are relatively uniform across the lobes (see Fig. 4), with no trend, that is, steepening, with the distance from the core.

We estimated that the magnetic field strength in NGC 3998 is $1 \mathrm{nT}(10 \mu \mathrm{G})$. We calculated the magnetic field strength in NGC 3998 following the equation from Worrall \& Birkinshaw (2006), assuming that the magnetic field and the particles satisfy energy equipartition. We also assumed a power-law distribution for the particles of the form $\mathrm{N}(\gamma) \propto \gamma^{-\alpha}$, with minimum and maximum Lorentz factors of $\gamma_{\min }=10$ and $\gamma_{\max }=10^{6}$, respectively. Following Brienza et al. (2016), we estimated that the source volume is $3.5 \times 10^{66} \mathrm{~cm}^{3}$ assuming a prolate ellipsoidal geometry with major and minor axes equal to the projected size of the source (i.e. $90^{\prime \prime}$ and $60^{\prime \prime}$ ). We further assumed that the particle energy content of the source is equally distributed between heavy particles and electrons.

With a magnetic field strength of $1 \mathrm{nT}(10 \mu \mathrm{G})$, a break frequency of $1400 \mathrm{MHz}$ would provide an upper limit on the age of the radio lobes of $38 \mathrm{Myr}$ at the redshift of NGC 3998. A note of caution is needed because the spectral index is derived only for low radio frequencies, that is, those below $1400 \mathrm{MHz}$. Our analysis still leaves the possibility that the emission is associated with the remnant of a recently switched off source where the break frequency, $v_{\text {off }}$, has not yet reached the low frequencies. Because of this, the age quoted above is to be considered as an upper limit.

This age can be compared with what was obtained from the analysis presented in Frank et al. (2016). The authors assumed an average velocity of propagation of the jets $\sim 1700 \mathrm{~km} \mathrm{~s}^{-1}$ (following the results for another low-power radio galaxy, Centaurus $\mathrm{A}$, by Croston et al. 2009) and derived timescales of a few times $10^{6}$ to at most $10^{7} \mathrm{yr}$ (see Frank et al. 2016 for the full discussion). These times are shorter than what was derived from the consideration on the spectral properties (which indeed was an upper limit), but they are consistent with no steepening at frequencies lower than $1400 \mathrm{MHz}$.

The spectral index derived for the lobes, the S-shape of the lobes, its similarity with the inner warp, and the presence of jet-like structures observed in the high-resolution LOFAR image (see Fig. 3) suggest a scenario in which the lobes are still actively fed by the central region or where the injection is only very recently stopped. However, more data are required to fully explain the morphology of the source, such as the bright nuclear region and the extreme low surface brightness of the lobes, and what this tells us about the evolution of the radio source.

\subsection{Sputtering radio source?}

Two possible scenarios can be considered to describe all the properties of the radio emission in NGC 3998: (i) although we have excluded that the lobes are old remnants, the overall radio structure could still be the result of intermittent fuelling; (ii) alternatively, it could be the result of intermittent flow that is the result of a strong interaction between a recently created jet and the surrounding rich medium, with the subsequent temporarily decollimation of the flow. The two scenarios are not necessarily disconnected and might even be complementary.

The idea that the activity is intermittent is supported by the variability of the core of NGC 3998; this has been pointed out by a number of authors (e.g. Kharb et al. 2012; Frank et al. 2016). It suggests that the fuelling may occur through discrete events. Based on the morphology and the spectral information, the timescales of the intermittent activity can be derived at least to first order. One cycle of activity has probably started very recently, as shown by the relatively bright core from which jets emerge (see e.g. Helmboldt et al. 2007 and Fig. 3). The previous cycle is traced by the low surface brightness lobes, and it happened at most a few dozen megayears ago, as discussed above. The adiabatic expansion of the lobes may be responsible for the low surface brightness of these structures. However, the lack of any gradient in the spectral index inside the lobe would require turbulence, for example, that is capable through diffuse shock acceleration (i.e. second-order Fermi acceleration) of re-accelerating old electrons but also of providing population mixing that would dilute any spectral gradient. This would help prevent the steepening of the spectral index due to radiative (and adiabatic) losses. This possibility has also been discussed in the case of other radio galaxies (e.g. Brienza et al. 2018, see also below). This process does not last more than a few dozen megayears, after which the lobes quickly fade away (Eilek 2014). On the other hand, the timescales of the intermittent activity, that is, the off time, should not be too long, otherwise, the spectrum would be steeper than what is observed. In this first scenario, the origin of the high contrast between the core and the lobes would therefore be due to the intermittent nature. However, the origin of the high turbulence that would dilute any spectral gradient is less clear.

The second scenario, strong interaction able to temporarily disrupt the jet, is motivated by the abrupt change in properties of the jets (transitioning to poorly collimated, low surface brightness plumes) as soon as they are outside the nuclear regions, and by the fact that the radio source resides in a gas rich galaxy. Based on the H I observations presented in Frank et al. (2016), we were unable to derive any sign of interaction between the jet and the gas. However, more recent observations of the ionised gas presented in Devereux (2018) may hold clues. Devereux (2018) reported the detection of broad permitted and forbidden emission lines using the high spatial resolution of STIS-HST. They ruled out the radiatively driven outflow scenario to explain the broad forbidden lines (e.g. [OIII] with a full width at halfmaximum, FWHM of $2185 \pm 265 \mathrm{~km} \mathrm{~s}^{-1}$ ) because the AGN is simply unable to provide sufficient radiation pressure. The possibility that the jet provides the energetics was ruled out by the author because the jet direction is perpendicular to the position angle of the STIS slit. However, at least the first part of the jet (e.g. the VLBI jet) would be included in the slit (which has a width of $0.1^{\prime \prime}$ ) and its power can, in principle, provide the energetics to produce the outflow. Furthermore, the region affected by the jet could be larger than the jet itself, for example, it mgith be a cocoon, see the simulations by Mukherjee et al. (2018a). If this is the case, it may support a strong interaction between jet and the ISM in the nuclear regions. Frank et al. (2016) have estimated a jet power of $P_{\text {jet }} \sim 1.64 \times 10^{42} \mathrm{erg} \mathrm{s}^{-1}$ derived using the relation given in Cavagnolo et al. (2010). This is likely to be a 
lower limit to the jet power because a substantial thermal component is likely present as well (see e.g. the case of IC 5063, Mukherjee et al. 2018a). Under these circumstances, the radio plasma would be able to drive the outflow.

Thus, in this second scenario, the low-power jet is not necessarily intermittent but is undergoing a strong interaction with the ISM. Because of this, the jet is temporarily trapped (and perhaps temporarily disrupted), piling up energy in the inner region of the galaxy, until it bursts and expands in the extended lobes. This interaction would decollimate the flow, and combined with the low velocity of the jet, would result in a turbulent flow, with an increased thermal component due to the entrainment of material from the surrounding medium. This would further slow down and diffuse the jet and cause the lobes to evolve into low surface brightness structures.

Interestingly, in NGC 3998 the lobes appear to have kept the imprint of the warp seen in the central sub-kiloparsec regions. This may further suggest that the lobes are still connected (and fed, even if at low rate) to the central region. This has been found to be the case in Centaurus A (see below), but high sensitivity combined with high spatial resolution is required to investigate the possible presence of a connecting channel in NGC 3998.

Thus, the morphology and characteristics of the radio source in NGC 3998 can be broadly described as resulting from intermittent emission from the central regions. This intermittency can be caused by genuine intermittent fuelling (but with a relatively short off time) or, more likely, from temporary disruption of the jet due to jet-ISM interaction (or a combination of the two), resulting in a kind of sputtering emission that would continue to feed the lobes, albeit perhaps at a low rate. In particular, the second scenario would also result in a strong decollimation of the jets, slowing down the flow in the lobes and causing their low surface brightness. This scenario would also better explain the presence of turbulence in the plasma flow.

\subsection{Relevance and connection with other sources}

The results on NGC 3998 are relevant in connection with the more general effort to understand the nature and evolution of low-power radio sources (including e.g. core galaxies, the so-called coreG Balmaverde \& Capetti 2006; Baldi \& Capetti 2009; galactic scale jets (GSJ), e.g. Webster in prep.; FR0 radio galaxies, Baldi et al. 2019; Capetti et al. 2019). Our observations in NGC 3998 confirm one of the possible scenarios put forward to explain the lack of large extended structures in these sources, and in particular, in FR0 galaxies: slower jets can be more subject to instabilities and entrainment, which causes their premature disruption and fading (Baldi et al. 2015). Thus, our results on NGC 3998 appear to support the scenario in which the lack of extended emission in these low-power sources would be due to the rapid fading of extended lobes. This would be due to a combination of interaction, temporary disruption of the plasma flow, and the large entrainment of thermal gas.

Extended radio sources and radio galaxies include cases that present a number of similarities with NGC 3998. Some examples have been discussed in Brienza et al. (2018). In particular, it is worth mentioning B2 $0258+35$ and Cen A as two cases of low-power radio galaxies with a very high surface brightness contrast between the central regions and the large-scale lobes. In both cases, and similarly to NGC 3998, the large lobes, despite the low-surface brightness, have a spectral index of actively fuelled structures (i.e. $\alpha \sim 0.7$ ). The spectral index of these two objects is also quite homogeneous across the lobes with no trend that could be connected with the ageing of the electrons (see
Brienza et al. 2018; Morganti et al. 1999; McKinley et al. 2018 for B2 $0258+35$ and Centaurus A, respectively). In B2 0258+35 the spectral index of the lobes does not show any significant curvature up to $6.6 \mathrm{GHz}$, suggesting even tighter requirements for the re-acceleration of the electrons in the lobes.

A low surface brightness large-scale jet connecting the bright central part with the large-scale lobes has been found in Centaurus A (Morganti et al. 1999; McKinley et al. 2018). This structure would ensure that the supplies of energy from the central galaxy to the lobes still occur (even if at a low rate, as suggested by their low surface brightness). This fuelling process likely also maintains the level of turbulence that is required for the in situ particle re-acceleration within the lobes, a process that would prevent the steepening of the radio spectrum.

Other objects with radio properties (and radio power) similar to NGC 3998 are the Seyfert galaxies Mrk 6 and NGC 6764 (Kharb et al. 2006, 2010). In these objects, the diffuse lobes or bubble also have a standard homogeneous spectral index (see Mrk 6, Kharb et al. 2006) and an S-shaped morphology, which have been explained as precessing jets. An interaction that temporarily disrupts the jet has also been suggested (see NGC 6764, Kharb et al. 2010).

In summary, the picture we assemble for NGC 3998 and other similar objects means that amorphous low surface brightness structures should not be taken by default as emission from remnant structures in low-power radio AGN. Furthermore, the proposed (sputtering) scenario has consequences for the morphology of the radio source. It would affect the way the source (and in particular, the large-scale emission) evolves and the way the source is classified.

Although objects such as NGC 3998 seem to be rare, the number of known low-power radio AGN with similar properties (albeit with a radio power more in the range of FRI radio galaxies) is now growing. In particular, the core dominance and the low surface brightness of the lobes (a few $\mathrm{mJy} \operatorname{arcmin}^{-2}$, i.e. at the limit of what is detectable with present-day radio telescopes) have been proposed to identify radio sources with intermittent activity (see e.g. Saripalli et al. 2012; Kuźmicz et al. 2017; Brienza et al. 2018; Jurlin et al., in prep.). For example, about $15 \%$ of the radio sources found in the LOFAR image of the Lockman Hole area (Brienza et al. 2017) are found to have a high core dominance (e.g. $S_{\text {core }} / S_{\text {tot }}>0.2$ ) and amorphous low surface brightness extended emission (see Jurlin et al., in prep.). Some of these sources have radio structures that are reminiscent of NGC 3998 structure. This means that they form not only an interesting, but also a relevant group of sources (see also Mingo et al. 2019 for a detailed discussion of the classification). The combination of the LOFAR images with images at $1400 \mathrm{MHz}$ becoming available, for common areas, from the APERTIF phased-array feed surveys on the WSRT, will allow us to obtain the necessary spectral index information.

\section{Conclusions}

We have presented new LOFAR $147 \mathrm{MHz}$ observations of the radio source associated with the galaxy NGC 3998. The new LOFAR observations have confirmed the properties of the radio structure observed at $1400 \mathrm{MHz}$ : the sources is core dominated with low surface brightness lobes. Most importantly, the observations have further shown that the lobes do not have an ultrasteep spectrum, as expected if they represented old remnant structures. Instead, they have a spectral index typical of still active structures (with an average value of $\alpha_{147 \mathrm{MHz}}^{1400 \mathrm{MHz}} \sim 0.6$ ). 
Furthermore, the spectral index is relative homogeneous across the lobes.

Based on the spectral properties, we estimated a (upper) limit to the age of the lobes (38 Myr) while conservative assumptions about the propagation of the jets suggest ages of the lobes between a few times $10^{6}$ to at most $10^{7} \mathrm{yr}$. The results suggest a scenario in which the lobes are still actively fed by the central region (or in which the injection stopped only very recently). Although the possibility of an intermittent fuelling (with a short off time) is supported by the variability of the core of NGC 3998 and may likely occur, the high contrast between the central regions and the large-scale lobes and the spectral properties suggests that the plasma is turbulent and that in situ re-acceleration occurs in the lobes. This can happen if the jet is temporarily disrupted by the interaction with a dense and rich circumnuclear medium.

Our findings for NGC 3998 would suggest that the lack of extended emission in low-power radio sources (e.g. FR0, core galaxies, and galactic scale jets) could be due to the rapid fading of extended lobes. This would be caused by a combination of interaction that temporarily disrupts the plasma flow and the large entrainment of thermal gas. Furthermore, the picture we assemble for NGC 3998 and other similar objects means that amorphous low surface brightness structures should not be taken by default as emission from remnant structures.

If the proposed scenario is correct, tracing the evolution of such low-power radio sources would be made more difficult by the relatively short timescale of their extended lobes if they quickly fade to very low surface brightness structure. However, a growing group of radio sources with properties similar to NGC 3998 is being identified in the deep (and high spatial resolution) surveys carried out at low frequencies by LOFAR (i.e. the LOFAR Two-metre Sky Survey, LoTSS (Shimwell et al. 2019). Thus, these sources (see e.g. Mingo et al. 2019; Jurlin et al., in prep.) can tell us about evolutionary paths of the radio plasma and/or about specific conditions of the ISM different from what is found in classical radio galaxies, that is, FRI and II. This helps completing our knowledge about the how radio emission shapes and is shaped by the surrounding medium.

Acknowledgements. We thank the anonymous referee for their helpful comments. The research leading to these results has received funding from the European Research Council under the European Union's Seventh Framework Programme (FP/2007-2013)/ERC Advanced Grant RADIOLIFE-320745. LOFAR, the Low Frequency Array designed and constructed by ASTRON, has facilities in several countries, that are owned by various parties (each with their own funding sources), and that are collectively operated by the Internationa LOFAR Telescope (ILT) foundation under a joint scientific policy. Basic research in radio astronomy at the U.S. Naval Research Laboratory is supported by 6.1 Base Funding.

\section{References}

Alam, S., Albareti, F. D., Allende Prieto, C., et al. 2015, ApJS, 219, 12 Baldi, R. D., \& Capetti, A. 2009, A\&A, 508, 603

Baldi, R. D., Capetti, A., \& Giovannini, G. 2015, A\&A, 576, A38

Baldi, R. D., Capetti, A., \& Giovannini, G. 2019, MNRAS, 482, 2294

Balmaverde, B., \& Capetti, A. 2006, A\&A, 447, 97

Best, P. N., Kauffmann, G., Heckman, T. M., et al. 2005, MNRAS, 362, 25

Bicknell, G. V., Dopita, M. A., Tsvetanov, Z. I., \& Sutherland, R. S. 1998, ApJ, 495, 680

Brienza, M., Godfrey, L., Morganti, R., et al. 2016, A\&A, 585, A29

Brienza, M., Godfrey, L., Morganti, R., et al. 2017, A\&A, 606, A98

Brienza, M., Morganti, R., Murgia, M., et al. 2018, A\&A, 618, A45

Briggs, D. 1995, PhD Thesis, New Mexico Institute of Mining and Technology

Cappellari, M., Emsellem, E., Krajnović, D., et al. 2011, MNRAS, 413, 813
Capetti, A., Baldi, R. D., Brienza, M., Morganti, R., \& Giovannini, G. 2019, A\&A, 631, A176

Cavagnolo, K. W., McNamara, B. R., Nulsen, P. E. J., et al. 2010, ApJ, 720, 1066 Ciotti, L., Ostriker, J. P., \& Proga, D. 2010, ApJ, 717, 708

Combes, F., García-Burillo, S., Casasola, V., et al. 2013, A\&A, 558, A124

Croston, J. H., Hardcastle, M. J., Kharb, P., Kraft, R. P., \& Hota, A. 2008, ApJ, 688,190

Croston, J. H., Kraft, R. P., Hardcastle, M. J., et al. 2009, MNRAS, 395, 1999

Devereux, N. 2018, MNRAS, 473, 2930

Eilek, J. A. 2014, New J. Phys., 16, 045001

Fabbiano, G., Paggi, A., Karovska, M., et al. 2018, ApJ, 865, 83

Fanaroff, B. L., \& Riley, J. M. 1974, MNRAS, 167, 31P

Frank, B. S., Morganti, R., Oosterloo, T., Nyland, K., \& Serra, P. 2016, A\&A, 592, A94

Gaspari, M., Ruszkowski, M., \& Oh, S. P. 2013, MNRAS, 432, 3401

Gaspari, M., Brighenti, F., \& Temi, P. 2015, A\&A, 579, A62

Harwood, J. J., Hardcastle, M. J., Croston, J. H., \& Goodger, J. L. 2013, MNRAS, 435, 3353

Heald, G., McKean, J., Pizzo, R., et al. 2010, ArXiv e-prints [arXiv:1008. 4693]

Heckman, T. M., \& Best, P. N. 2014, ARA\&A, 52, 589

Heesen, V., Croston, J. H., Morganti, R., et al. 2018, MNRAS, 474, 5049

Helmboldt, J. F., Taylor, G. B., Tremblay, S., et al. 2007, ApJ, 658, 203

Husemann, B., Scharwächter, J., Davis, T. A., et al. 2019, A\&A, 627, A53

Intema, H. T., Jagannathan, P., Mooley, K. P., \& Frail, D. A. 2017, A\&A, 598, A78

Kharb, P., O’Dea, C. P., Baum, S. A., Colbert, E. J. M., \& Xu, C. 2006, ApJ, 652, 177

Kharb, P., Hota, A., Croston, J. H., et al. 2010, ApJ, 723, 580

Kharb, P., Capetti, A., Axon, D. J., et al. 2012, AJ, 143, 78

Krajnović, D., Emsellem, E., Cappellari, M., et al. 2011, MNRAS, 414, 2923

Kuźmicz, A., Jamrozy, M., Kozieł-Wierzbowska, D., \& Weżgowiec, M. 2017, MNRAS, 471, 3806

Laing, R. A., \& Bridle, A. H. 2014, MNRAS, 437, 3405

Massaglia, S., Bodo, G., Rossi, P., Capetti, S., \& Mignone, A. 2016, A\&A, 596, A12

Mauch, T., \& Sadler, E. M. 2007, MNRAS, 375, 931

McKinley, B., Tingay, S. J., Carretti, E., et al. 2018, MNRAS, 474, 4056

Mingo, B., Croston, J. H., Hardcastle, M. J., et al. 2019, MNRAS, 488, 2701

Morganti, R. 2017, Nat. Astron., 1, 596

Morganti, R., Oosterloo, T. A., Reynolds, J. E., Tadhunter, C. N., \& Migenes, V. 1997, MNRAS, 284, 541

Morganti, R., Killeen, N. E. B., Ekers, R. D., \& Oosterloo, T. A. 1999, MNRAS, 307,750

Morganti, R., Oosterloo, T., Oonk, J. B. R., Frieswijk, W., \& Tadhunter, C. 2015, A\&A, 580, A1

Mukherjee, D., Bicknell, G. V., Wagner, A. E. Y., Sutherland, R. S., \& Silk, J. 2018a, MNRAS, 479, 5544

Mukherjee, D., Wagner, A. Y., Bicknell, G. V., et al. 2018b, MNRAS, 476, 80

Negri, A., Pellegrini, S., \& Ciotti, L. 2015, MNRAS, 451, 1212

Nyland, K., Young, L. M., Wrobel, J. M., et al. 2016, MNRAS, 458, 2221

Nyland, K., Young, L. M., Wrobel, J. M., et al. 2017, MNRAS, 464, 1029

Offringa, A. R., de Bruyn, A. G., Biehl, M., et al. 2010, MNRAS, 405, 155

Offringa, A. R., van de Gronde, J. J., \& Roerdink, J. B. T. M. 2012, A\&A, 539, A95

Offringa, A. R., McKinley, B., Hurley-Walker, N., et al. 2014, MNRAS, 444, 606 Pandey, V. N., van Zwieten, J. E., de Bruyn, A. G., \& Nijboer, R. 2009, in The Low-Frequency Radio Universe, eds. D. J. Saikia, D. A. Green, Y. Gupta, \& T. Venturi, ASP Conf. Ser., 407, 384

Parma, P., Murgia, M., de Ruiter, H. R., \& Fanti, R. 2002, New A Rev., 46, 313

Rodríguez-Ardila, A., Prieto, M. A., Mazzalay, X., et al. 2017, MNRAS, 470, 2845

Sabater, J., Best, P. N., Hardcastle, M. J., et al. 2019, A\&A, 622, A17

Sánchez-Gallego, J. R., Knapen, J. H., Wilson, C. D., et al. 2012, MNRAS, 422, 3208

Saripalli, L., Subrahmanyan, R., Thorat, K., et al. 2012, ApJS, 199, 27

Scaife, A. M. M., \& Heald, G. H. 2012, MNRAS, 423, L30

Serra, P., Oosterloo, T., Morganti, R., et al. 2012, MNRAS, 422, 1835

Shimwell, T. W., Tasse, C., Hardcastle, M. J., et al. 2019, A\&A, 622, A1

Storchi-Bergmann, T., \& Schnorr-Müller, A. 2019, Nat. Astron., 3, 48

van Haarlem, M. P., Wise, M. W., Gunst, A. W., et al. 2013, A\&A, 556, A2

van Weeren, R. J., Williams, W. L., Hardcastle, M. J., et al. 2016, ApJS, 223, 2

Williams, W. L., van Weeren, R. J., Röttgering, H. J. A., et al. 2016, MNRAS, 462, 1910

Worrall, D. M., \& Birkinshaw, M. 2006, in Multiwavelength Evidence of the Physical Processes in Radio Jets, ed. D. Alloin, 693, 39

Younes, G., Ptak, A., Ho, L. C., et al. 2019, ApJ, 870, 73

Yuan, F., \& Narayan, R. 2014, ARA\&A, 52, 529 